TRANSACTIONS OF THE

AMERICAN MATHEMATICAL SOCIETY

Volume 359, Number 4, April 2007, Pages 1471-1484

S 0002-9947(06)04203-6

Article electronically published on October 17, 2006

\title{
COMPACTNESS PROPERTIES OF SOBOLEV IMBEDDINGS FOR REARRANGEMENT INVARIANT NORMS
}

\author{
GUILLERMO P. CURBERA AND WERNER J. RICKER
}

\begin{abstract}
Compactness properties of Sobolev imbeddings are studied within the context of rearrangement invariant norms. Attention is focused on the extremal situation, namely, when the imbedding is considered as defined on its optimal Sobolev domain (with the range space fixed). The techniques are based on recent results which reduce the question of boundedness of the imbedding to boundedness of an associated kernel operator (of just one variable).
\end{abstract}

\section{INTRODUCTION}

The Rellich-Kondrachov theorem on compactness of the classical Sobolev imbedding (for suitable $\Omega \subset \mathbb{R}^{n}$ ) asserts that the imbedding $W_{0}^{1, p}(\Omega) \hookrightarrow L^{q}(\Omega)$ is compact for $1 \leq q<n p /(n-p)$ whenever $1 \leq p<n$. In the case $q=n p /(n-p)$, although Sobolev's theorem ensures boundedness, the imbedding is not compact. Equivalently, if $n^{\prime}$ is the conjugate index of $n$ and we fix $n^{\prime} \leq q<\infty$, then there is compactness of the imbedding whenever $p \in(n q /(n+q), n)$, but not at the endpoint $p=n q /(n+q)$. However, for $1 \leq q<n^{\prime}$, the imbedding is compact for all $p \in[1, n)$. Note that there is always compactness for $p \geq n$. So, for the imbedding $W_{0}^{1, p}(\Omega) \hookrightarrow L^{q}(\Omega)$, if we fix the range space to be some $L^{q}(\Omega)$ smaller than $L^{n^{\prime}}(\Omega)$, then the imbedding remains compact as long as the domain space $W_{0}^{1, p}(\Omega)$ does not reach the space $W_{0}^{1, n q /(n+q)}(\Omega)$ which is "too large" (i.e. the endpoint $n q /(n+q)$ is avoided). But, if we fix the range space to be some $L^{q}(\Omega)$ larger than $L^{n^{\prime}}(\Omega)$, then the imbedding is compact for all domain spaces $W_{0}^{1, p}(\Omega)$ (since it is so for $W_{0}^{1,1}(\Omega)$ ), i.e. no endpoint occurs. Donaldson and Trudinger (see [9] and [1, Ch. VIII]) considered existence and compactness of the imbedding within the class of Orlicz-Sobolev spaces (the class of Orlicz spaces properly contains the $L^{p}$-spaces). Our aim is to study the compactness/noncompactness phenomena described above within the framework of Sobolev inequalities for norms even more general than Orlicz norms (indeed, for arbitrary rearrangement invariant norms). The advantage of using a richer family of spaces/norms is well illustrated by a celebrated result of Trudinger 20. where, by using the scale of Orlicz spaces, a sharp range space can be found

Received by the editors January 3, 2005.

2000 Mathematics Subject Classification. Primary 46E35, 46E30; Secondary 47G10.

Key words and phrases. Sobolev imbeddings, compactness, rearrangement invariant spaces, optimal domains.

The authors gratefully acknowledge the support of the Katholische Universität Eichstätt-Ingolstadt (Germany) and D.G.I. \#BFM2003-06335-C03-01 (Spain).

The results of this paper were presented at the 7th International Conference on Harmonic Analysis and Partial Differential Equations, held at El Escorial, Spain, in June 2004.

(C)2006 American Mathematical Society 
for the Sobolev imbedding. Indeed, while $W_{0}^{1, n}(\Omega) \hookrightarrow L^{q}(\Omega)$ for all $1 \leq q<\infty$, but not for $q=\infty$, we have that $W_{0}^{1, n}(\Omega) \hookrightarrow L^{\varphi}(\Omega)$, where $\varphi(t)=\exp \left(t^{n^{\prime}}\right)-1$, with $L^{\infty}(\Omega) \subsetneq L^{\varphi}(\Omega) \subsetneq L^{q}(\Omega)$ for all $1 \leq q<\infty$. We will use this advantage in order to get finer results in the study of compactness/noncompactness of the Sobolev imbedding.

More precisely, let $\Omega \subset \mathbb{R}^{n}$ be a bounded domain having Lebesgue measure one. In [1], Edmunds, Kerman and Pick considered rearrangement invariant (briefly, r.i.) spaces $X, Y$ on $[0,1]$ and a generalized Sobolev inequality $\left\|u^{*}\right\|_{X} \leq C\left\||\nabla u|^{*}\right\|_{Y}$ for $u \in C_{0}^{1}(\Omega)$, where $u^{*}$ and $|\nabla u|^{*}$ are, respectively, the decreasing rearrangements of $u$ and of the norm of its gradient. Defining $X(\Omega):=\left\{u: \Omega \rightarrow \mathbb{R}: u^{*} \in X\right\}$ and setting $\|u\|_{X(\Omega)}:=\left\|u^{*}\right\|_{X}$ (which is a norm because $X$ is r.i.), the above inequality becomes

$$
\|u\|_{X(\Omega)} \leq C\|\mid \nabla u\|_{Y(\Omega)}, \quad u \in C_{0}^{1}(\Omega) .
$$

By a generalized Poincaré inequality due to Cianchi and Pick, [5, Lemma 4.2], there is a constant $M>0$ (depending only on $\Omega$ and $n$ ) such that, if $Y$ is any r.i. space, then $\|u\|_{Y(\Omega)} \leq M\|\mid \nabla u\|_{Y(\Omega)}$ for $u \in C_{0}^{1}(\Omega)$. Hence, (1.1) is equivalent, with a different constant $C$, to $\|u\|_{X(\Omega)} \leq C\left(\|u\|_{Y(\Omega)}+\|\mid \nabla u\|_{Y(\Omega)}\right)$ for $u \in C_{0}^{1}(\Omega)$. Defining the Sobolev space $W_{0}^{1} X(\Omega)$ as the closure of $C_{0}^{1}(\Omega)$ with respect to the norm $\|u\|_{W_{0}^{1} X(\Omega)}:=\|u\|_{X(\Omega)}+\|\mid \nabla u\|_{X(\Omega)}$ (see e.g. [5], 12], 19]), it follows that (1.1) is equivalent to boundedness of the inclusion

$$
j: W_{0}^{1} Y(\Omega) \hookrightarrow X(\Omega) .
$$

Edmunds, Kerman and Pick studied the boundedness of (1.2) by showing that (1.1) is equivalent to boundedness, from $Y$ to $X$, of the kernel operator $T$ associated with Sobolev's inequality, namely $T f(t):=\int_{t}^{1} f(s) s^{-1 / n^{\prime}} d s$ for $t \in[0,1]$. That is, $\|T f\|_{X} \leq K\|f\|_{Y}$, [11, Theorem 6.1]. We will investigate compactness of the Sobolev imbedding (1.2) via the associated kernel operator $T$, typically acting on classical function spaces, the advantage being that the general theory of such operators is well understood. In this regard, we consider the optimal (=maximal) lattice domain for $T$ with values in an r.i. space $X$. The maximality is to be understood in the following sense: there is a continuous linear extension of $T$ from $[T, X]$ into $X$, and if $T$ has any continuous linear extension from some Banach function space $F$ (on $[0,1]$ ) into $X$, then $F$ is continuously included in $[T, X]$. An equivalent description of the optimal domain $[T, X]$ can be formulated. The kernel

$$
K(t, s):=s^{-1 / n^{\prime}} \chi_{[t, 1]}(s), \quad(t, s) \in[0,1] \times(0,1]
$$

generates the operator $T$. Since $K$ is nonnegative, $[T, X]=\{f: T|f| \in X\},[7$, Proposition 5.2]. Moreover, $K$ satisfies the conditions of [7, Theorem 5.12] and so

$$
[T, X]=\left(L^{1}\left(s^{1 / n} d s\right), L^{1}\left(s^{-1 / n^{\prime}} d s\right)\right)_{X},
$$

where this last interpolation space is obtained via the $K$-functional and the r.i. norm of $X$; see [3, V.1].

In general, the space $[T, X]$ is not r.i., in which case it becomes unclear what the corresponding "space" $W_{0}^{1}[T, X](\Omega)$ should be. Thus, we are naturally lead to consider the largest r.i. space continuously imbedded in $[T, X]$, which we denote by $[T, X]^{r i}$; for the existence of $[T, X]^{r i}$ we refer to $[4$, Proposition 2.2.6]. That 
is, $[T, X]^{r i}$ is the maximal r.i. space on $[0,1]$ to which $T$ can be extended as a continuous linear operator with values still in $X$. Hence,

$$
j: W_{0}^{1}[T, X]^{r i}(\Omega) \hookrightarrow X(\Omega)
$$

is bounded and this imbedding is optimal, that is, for the fixed range space $X(\Omega)$ we cannot replace $[T, X]^{r i}$ by any larger r.i. space. Equivalently, $\|u\|_{X(\Omega)} \leq$ $C\||\nabla u|\|_{[T, X]^{r i}(\Omega)}$ for $u \in C_{0}^{1}(\Omega)$, with the bound optimal: the $[T, X]^{r i}$ norm cannot be replaced by any smaller r.i. norm. In this sense we say that $W_{0}^{1}[T, X]^{r i}(\Omega)$ is the optimal Sobolev domain corresponding to the fixed range space $X(\Omega)$.

It turns out that compactness/noncompactness of the Sobolev imbedding $j: W_{0}^{1}[T, X]^{r i}(\Omega) \hookrightarrow X(\Omega)$ is intimately connected to that of the associated kernel operator $T:[T, X]^{r i} \rightarrow X$. This is rather interesting, given that the extended operator $T:[T, X] \rightarrow X$ is never compact, [8, Propositions 2.2(c) and 3.6(d)] and [7. Proposition 5.2(a)]. So, questions concerning compactness of $j$ on its optimal Sobolev domain can be treated via a kernel operator acting on an r.i. space of functions of one variable. This warrants a detailed investigation of the compactness properties of $T:[T, X]^{r i} \rightarrow X$, which is carried out in Section 2 .

Section 3 is devoted to the study of compactness properties of $j: W_{0}^{1}[T, X]^{r i}(\Omega)$ $\hookrightarrow X(\Omega)$. Since $j: W_{0}^{1, p}(\Omega) \hookrightarrow X(\Omega)$ is compact whenever $p>n$, a similar feature occurs as in the Rellich-Kondrachov theorem. Namely, whenever $X(\Omega)$ is "appropriately smaller" than $L^{n^{\prime}, \infty}(\Omega)$, then there is compactness of the Sobolev imbedding as long as we do not reach a domain space which is "too large". Surely compactness fails at the optimal Sobolev domain. On the other hand, whenever $X(\Omega)$ is "appropriately larger" than $L^{n^{\prime}, \infty}(\Omega)$, then the Sobolev imbedding is compact for every domain space, since it is so for $W_{0}^{1,1}(\Omega)=W_{0}^{1} L^{1}(\Omega)$.

Special emphasis has been given to the study of relevant examples arising from spaces which occur in classical analysis. For instance, we consider the Lorentz $L^{p, q}$ spaces, the Lorentz $\Lambda_{\varphi}$ and Marcinkiewicz $M_{\varphi}$ spaces (in particular, $L \log L$ and $\left.L_{\text {exp }}\right)$, and, more generally, the Lorentz-Zygmund spaces $L^{p, q}(\log L)^{\alpha}$. Particular attention is paid to the subclass of spaces, $\operatorname{Exp} L^{p}$, consisting of those functions which are exponentially $p$-integrable, which includes the Orlicz space considered by Trudinger.

\section{Preliminaries}

An r.i. space $X$ on $[0,1]$ is a Banach space of integrable functions on $[0,1]$ which contains the simple functions, has the property that $g \in X$ with $\|g\| \leq\|f\|$ whenever $f \in X$ and $g$ satisfies $|g| \leq|f|$, satisfies the Fatou property, and has the property that whenever $f \in X$ and $g$ is equimeasurable with $f$, then $g \in X$ and $\|g\|=\|f\|$. In particular, if $f^{*}$ is the decreasing rearrangement of $f \in X$, then $f^{*} \in X$ with $\left\|f^{*}\right\|=\|f\|$, 3, I.1.1 and II.4.1]. Hence, $L^{\infty}([0,1]) \subset X \subset L^{1}([0,1])$ continuously.

Let $\varphi$ be an increasing, concave function on $[0,1]$ with $\varphi(0)=0$. The Lorentz space $\Lambda_{\varphi}$ associated to $\varphi$ is defined by

$$
\Lambda_{\varphi}:=\left\{f:\|f\|_{\Lambda_{\varphi}}:=\int_{0}^{1} f^{*}(s) d \varphi(s)<\infty\right\},
$$


and the Marcinkiewicz space $M_{\varphi}$ associated to $\varphi$ is given by

$$
M_{\varphi}:=\left\{f:\|f\|_{M_{\varphi}}:=\sup _{0<t \leq 1} \frac{1}{\varphi(t)} \int_{0}^{t} f^{*}(s) d s<\infty\right\} .
$$

Both are r.i. spaces, [17, p. 112]. We also consider such spaces for a quasiconcave function $\varphi$ (concavity is replaced with $\varphi(t) / t$ decreasing) since, in this case, there is an equivalent concave function, [17, p. 49]. If two functions are equivalent, [17, p. 48], then the corresponding Lorentz and Marcinkiewicz spaces are isomorphic.

The fundamental function $\varphi_{X}$ of an r.i. space $X$ is defined by $\varphi_{X}(t):=\left\|\chi_{[0, t]}\right\|_{X}$. It is increasing, quasiconcave with $\varphi_{X}\left(0^{+}\right):=\lim _{t \rightarrow 0^{+}} \varphi_{X}(t) \geq 0$. We may assume $\varphi_{X}$ is concave, [3, II.5.11]. The space $\Lambda_{X}:=\Lambda_{\varphi}\left(\right.$ for $\left.\varphi:=\varphi_{X}\right)$ is the smallest r.i. space having fundamental function $\varphi_{X}$. The space $M_{X}:=M_{\varphi}\left(\right.$ for $\left.\varphi(t):=t / \varphi_{X}(t)\right)$ is the largest r.i. space having fundamental function $\varphi_{X}$. By a result of Semenov, $\Lambda_{X} \hookrightarrow X \hookrightarrow M_{X}$, [17, pp. 118-119].

For properties of the lower dilation exponent,

$$
\gamma_{\varphi}:=\lim _{t \rightarrow 0^{+}} \frac{\log \sup _{s} \varphi(s t) / \varphi(s)}{\log t},
$$

of an increasing concave function $\varphi$, see [17, p. 54]. If $\gamma_{\varphi}>0$, the expression

$$
\sup _{0<t \leq 1} \frac{t}{\varphi(t)} f^{*}(t)
$$

is equivalent to the norm in $M_{\varphi}$; see [17, pp. 114-115 and 56-57]. Setting $\bar{\varphi}(t):=$ $t / \varphi(t)$, a sufficient condition for $\gamma_{\varphi}>0$ is that $\bar{\varphi}(t) / t^{\varepsilon}$ is decreasing near zero for some $0<\varepsilon<1$. For the upper dilation exponent $\delta_{\varphi}$, see also [17, p. 54].

We repeatedly use the following facts concerning the optimal domain $[T, X]$ of the operator $T$ acting on an r.i. space $X$ with fundamental function $\varphi_{X}$.

Remark 2.1. (a) The space $L^{1}\left(t^{-1 / n^{\prime}} \varphi_{X}(t)\right)$ is contained in $[T, X]$.

(b) If $t^{-1 / n^{\prime}} \varphi_{X}(t)$ is decreasing, then the Lorentz space $\Lambda_{\Theta}$, with $\Theta(t):=$ $t^{1 / n} \varphi_{X}(t)$, is the largest r.i. space inside $L^{1}\left(t^{-1 / n^{\prime}} \varphi_{X}(t)\right)$. Consequently, $\Lambda_{\Theta} \subset$ $[T, X]^{r i}$.

The proof of these facts relies on an alternative description of the optimal domain space $[T, X]$ in terms of the $L^{1}$-space of an associated vector measure $\nu_{X}$. Indeed, if $A$ is a Borel set in $[0,1]$, then $T\left(\chi_{A}\right) \in X$ and the $X$-valued set function $\nu_{X}$ : $A \mapsto \nu_{X}(A):=T\left(\chi_{A}\right)$ is countably additive, i.e. it is a vector measure in $X$. Let $L^{1}\left(\nu_{X}\right)$ denote the space of all $\nu_{X}$-integrable functions, equipped with the topology of convergence in mean (see [7). Under certain conditions, the space $L^{1}\left(\nu_{X}\right)$ coincides with $[T, X]$, 7, Proposition 5.2]. With this result, we are able to apply the theory and techniques used in our study of $L^{1}\left(\nu_{X}\right)$, [7, to the space $[T, X]$.

In this setting, statement (a) follows from $L^{1}\left(t^{-1 / n^{\prime}} \varphi_{X}(t)\right)=L^{1}\left(\left|\nu_{X}\right|\right) \subset L^{1}\left(\nu_{X}\right)$ $\subset[T, X]$, where $\left|\nu_{X}\right|$ is the variation measure of $\nu_{X}$. The equality follows from Proposition 3.1(a) of [8], the first inclusion from Theorem 4.1 of [18] and the second inclusion from Proposition 5.2(a) of [7].

The first part of (b) is Proposition 4.7 and Remark 4.8 of 8 together with $L^{1}\left(t^{-1 / n^{\prime}} \varphi_{X}(t)\right)=L^{1}\left(\left|\nu_{X}\right|\right)$. The second part follows from $\Lambda_{\Theta} \subset[T, X]$ and the fact that $\Lambda_{\Theta}$ is r.i. 


\section{Compactness PROPERTIES OF THE ASSOCIATED KERNEL OPERATOR}

In this section we establish criteria for determining both noncompactness (cf. Theorem 3.7) and compactness (cf. Theorem 3.9) of the $X$-valued kernel operator $T$ when considered as acting on its optimal r.i. domain $[T, X]^{r i}$. In view of the fact that $T: X \rightarrow X$ is "almost always" compact (i.e. under very general conditions on the r.i. space $X,[8$, Remark 3.7]) and that $T:[T, X] \rightarrow X$ is never compact (cf. Introduction), it is a priori unclear what form such results should take. The two main theorems mentioned above (also of independent interest) will be needed in the following section dealing with compactness properties of the Sobolev imbedding $j: W_{0}^{1}[T, X]^{r i}(\Omega) \hookrightarrow X(\Omega)$. We begin by identifying the spaces $[T, X]^{r i}$ for some classical r.i. spaces $X$.

Example 3.1. For $X=L^{p}([0,1])$ and $n^{\prime}<p<\infty$, the optimal r.i. domain $\left[T, L^{p}\right]^{r i}$ is the Lorentz $L^{p, q}$-space $L^{p_{0}, p}([0,1])$, where $p_{0}:=n p /(n+p)$, [19, Theorem 3.20]. When $p=\infty$ we have $\left[T, L^{\infty}\right]=L^{1}\left(s^{-1 / n^{\prime}}\right)$, [8, Proposition 2.1(d)], and hence, direct computation shows that $\left[T, L^{\infty}\right]^{r i}=L^{n, 1}([0,1])$.

Concerning Example 3.1. for a given $p_{0}$ we have $p=n p_{0} /\left(n-p_{0}\right)$. Note that this is precisely the exponent corresponding to $p_{0}$ in the classical Sobolev inequality $\|u\|_{p} \leq C\||\nabla u|\|_{p_{0}}$. Hence, this Sobolev inequality is actually sharpened, since $\left[T, L^{p}\right]^{r i}=L^{p_{0}, p}$ implies that $\|u\|_{p} \leq C\||\nabla u|\|_{p_{0}, p}$, with $\||\nabla u|\|_{p_{0}, p} \leq$ $\||\nabla u|\|_{p_{0}, p_{0}}=\||\nabla u|\|_{p_{0}}\left(\right.$ as $\left.p>p_{0}\right)$. This sharpening is optimal within the class of r.i. norms.

Example 3.2. Let $X=\Lambda_{\varphi}$ be a Lorentz $\Lambda$-space with $t^{-1 / n^{\prime}} \varphi(t)$ decreasing. Then $\left[T, \Lambda_{\varphi}\right]^{r i}$ is the Lorentz $\Lambda$-space $\Lambda_{\Theta}$, where $\Theta(t):=t^{1 / n} \varphi(t)$; see Remark 2.1)(b) and [8. Corollary 4.3]. Consider the Lorentz space $\Lambda_{X}$ corresponding to an r.i. space $X$ with a fundamental function $\varphi_{X}$ for which $t^{-1 / n^{\prime}} \varphi_{X}(t)$ is decreasing. Then $\left[T, \Lambda_{X}\right]^{r i}=\Lambda_{\Theta_{X}}$, for $\Theta_{X}(t):=t^{1 / n} \varphi_{X}(t)$. In particular, for the Lorentz spaces $L^{p, 1}([0,1])$ the condition is $n^{\prime} \leq p<\infty$ and we have $\left[T, L^{p, 1}\right]^{r i}=L^{p_{0}, 1}([0,1])$, with $p_{0}:=n p /(n+p)$.

Example 3.3. Let $X=M_{\varphi}$ be a Marcinkiewicz space for which $1 / n<\gamma_{\varphi} \leq \delta_{\varphi}<1$ holds. Then $\left[T, M_{\varphi}\right]^{r i}$ is the Marcinkiewicz space $M_{\Psi}$, where $\Psi(t):=t^{-1 / n} \varphi(t)$, 8 , Theorem 5.7]. For the Marcinkiewicz space $M_{X}$ associated to an r.i. space $X$ (with fundamental function $\varphi_{X}$ ), the above condition becomes $0<\underline{\lambda}_{X} \leq \bar{\lambda}_{X}<1 / n^{\prime}$, where $\underline{\lambda}_{X}, \bar{\lambda}_{X}$ are the fundamental indices of $X,\left[3\right.$, p. 177]. Moreover, $\left[T, M_{X}\right]^{r i}=$ $M_{\Psi_{X}}$, for $\Psi_{X}(t):=t^{1 / n^{\prime}} / \varphi_{X}(t)$ (which has fundamental function $t^{1 / n} \varphi_{X}(t)$ ). In

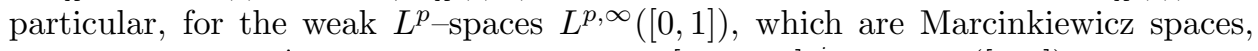
the condition is $n^{\prime}<p<\infty$ and we have $\left[T, L^{p, \infty}\right]^{r i}=L^{p_{0}, \infty}([0,1])$, with $p_{0}:=$ $n p /(n+p)$.

Set $p=n^{\prime}$ in Example 3.2 then $\left[T, L^{n^{\prime}, 1}\right]^{r i}=L^{1}([0,1])$. Moreover, Corollary 4.3 and Proposition 3.1(a) of $\left[8\right.$ imply that $\left[T, L^{n^{\prime}, 1}\right]=L^{1}([0,1])$. So, for $X=$ $L^{n^{\prime}, 1}([0,1])$, the optimal domain $[T, X]$ is itself already r.i. and hence, coincides with $[T, X]^{r i}$. The following result characterizes this phenomenon.

Theorem 3.4. Let $X$ be an r.i. space. The optimal domain $[T, X]$ is itself r.i. (i.e. $\left.[T, X]=[T, X]^{r i}\right)$ if and only if $X=L^{n^{\prime}, 1}([0,1])$.

Proof. Assume that $[T, X]$ is r.i.. Then $[T, X] \subset L^{1}([0,1])$. Let $f \in L^{1}([0,1])$. Consider the dilation operator $D_{2}$ defined by $D_{2} h(t):=h(2 t) \chi_{[0,1 / 2]}$. Then, $D_{2} f \in$ 
$L^{1}([0,1])$. Let $g(t):=D_{2} f(1-t)$. Then $g=0$ on $[0,1 / 2)$, and so

$$
\int_{0}^{1}|g(t)| t^{-1 / n^{\prime}} \varphi_{X}(t) d t<\infty
$$

that is, $g \in L^{1}\left(t^{-1 / n^{\prime}} \varphi_{X}(t)\right)$. By Remark $2.1\left(\right.$ a),$L^{1}\left(t^{-1 / n^{\prime}} \varphi_{X}(t)\right) \subset[T, X]$ and thus, $g \in[T, X]$. But, $[T, X]$ is r.i. with $g$ and $D_{2} f$ equimeasurable. So, $D_{2} f \in[T, X]$. Since dilation operators are bounded on r.i. spaces, [3, III.5.11], for $D_{1 / 2} h(t)=$ $h(t / 2)$ with $0 \leq t \leq 1$, we have $f=D_{1 / 2} D_{2} f \in[T, X]$. Hence, $L^{1}([0,1]) \subset[T, X]$, and so $[T, X]=L^{1}([0,1])$. Accordingly, $[T, X]$ is an AL-space, i.e. the norm is additive in the cone of positive functions. So, [8, Theorem 4.2] implies that $X$ must be a Lorentz $\Lambda$-space. In particular, $X$ has absolutely continuous norm, so by [7, Proposition 5.2.(c)], 6. Proposition 2], and [8, Proposition 3.1(a)] it follows that $L^{1}\left(t^{-1 / n^{\prime}} \varphi_{X}(t)\right)=L^{1}([0,1])$, and so the weights of both $L^{1}$-spaces are equivalent. Hence, $\varphi_{X}(t)$ is equivalent to $t^{1 / n^{\prime}}$. The Lorentz $\Lambda$-space with fundamental function (equivalent to) $t^{1 / n^{\prime}}$ is precisely $L^{n^{\prime}, 1}([0,1])$.

Remark 3.5. Since $L^{1}([0,1])$ is the largest possible r.i. space, it follows for every r.i. space $X$ with $L^{n^{\prime}, 1}([0,1]) \subset X$ that $[T, X]^{r i}=L^{1}([0,1])$. In particular, $\left[T, L^{p}\right]^{r i}=$ $L^{1}([0,1])$ for $1 \leq p \leq n^{\prime}$. More generally, $\left[T, L^{p, q}\right]^{r i}=L^{1}([0,1])$ for $1<p \leq n^{\prime}$ and $1 \leq q \leq \infty$, or for $p=q=1$.

The behaviour of the function $t \mapsto t^{-1 / n^{\prime}} \varphi_{X}(t)$ is of major importance concerning and connecting the compactness/noncompactness properties of both the kernel operator $T:[T, X]^{r i} \rightarrow X$ and the Sobolev imbedding $j: W_{0}^{1}[T, X]^{r i}(\Omega) \hookrightarrow X(\Omega)$.

Proposition 3.6. Let $X$ be an r.i. space with $t^{-1 / n^{\prime}} \varphi_{X}(t)$ decreasing. Let $\Theta(t):=$ $t^{1 / n} \varphi_{X}(t)$. Then $T: \Lambda_{\Theta} \rightarrow X$ is noncompact.

Proof. Let $\left(t_{k}\right)$ be a sequence in $(0,1]$ decreasing to zero and define $\alpha_{k}:=\left\|\chi_{\left(0, t_{k}\right]}\right\|_{\Lambda_{\ominus}}$, so that for $f_{k}:=\left(1 / \alpha_{k}\right) \chi_{\left(0, t_{k}\right]}$ we have $\left\|f_{k}\right\|_{\Lambda_{\ominus}}=1$. Direct computation shows that $T f_{k}(t)=\int_{t}^{1} f_{k}(s) s^{-1 / n^{\prime}} d s$ is equal to $\left(n / \alpha_{k}\right) \max \left\{t_{k}^{1 / n}-t^{1 / n}, 0\right\}$, which is a decreasing function.

Suppose $\left(T f_{k}\right)$ has a subsequence, again denoted by $\left(T f_{k}\right)$, which converges in $X$. Since the function $T f_{k}$ is supported in $\left(0, t_{k}\right]$, the sequence $\left(t_{k}\right)$ decreases to zero, and since convergence in $X$ implies - for a subsequence - convergence a.e., 3, I.1.7], it follows that the limit is the zero function. To obtain a contradiction, we show that $\left\|T f_{k}\right\|_{X}$ does not converge to zero. Since $\varphi_{X}(t) / t$ is decreasing, we have

$$
\begin{aligned}
\left\|T f_{k}\right\|_{X} \geq\left\|T f_{k}\right\|_{M_{X}} & =\sup _{0<t \leq 1} \frac{\varphi_{X}(t)}{t} \int_{0}^{t}\left(T f_{k}\right)^{*}(s) d s \\
& =\sup _{0<t \leq 1} \frac{\varphi_{X}(t)}{t} \int_{0}^{t} \frac{n}{\alpha_{k}} \max \left\{t_{k}^{1 / n}-s^{1 / n}, 0\right\} d s \\
& =\frac{n}{\alpha_{k}} \sup _{0<t \leq t_{k}} \frac{\varphi_{X}(t)}{t} \int_{0}^{t}\left(t_{k}^{1 / n}-s^{1 / n}\right) d s \\
& \geq \frac{n}{\alpha_{k}} \frac{\varphi_{X}\left(t_{k}\right)}{t_{k}} \int_{0}^{t_{k}}\left(t_{k}^{1 / n}-s^{1 / n}\right) d s \\
& =\frac{n}{\alpha_{k}} \frac{\varphi_{X}\left(t_{k}\right) t_{k}^{1 / n}}{n+1} .
\end{aligned}
$$


Since $\alpha_{k}=\int_{0}^{1}\left(\chi_{\left(0, t_{k}\right]}\right)^{*}(t) d \Theta(t)=\Theta\left(t_{k}\right)=t_{k}^{1 / n} \varphi_{X}\left(t_{k}\right)$, it follows that $\left\|T f_{k}\right\|_{\Lambda_{\Theta}} \geq$ $n /(n+1)$ for every $k \geq 1$. Therefore, $T$ is noncompact.

An immediate consequence is the following result.

Theorem 3.7. Let $X$ be an r.i. space with $t^{-1 / n^{\prime}} \varphi_{X}(t)$ decreasing. Then $T:[T, X]^{r i}$ $\rightarrow X$ is not compact.

Proof. Remark 2.1(b) shows that $\Lambda_{\Theta} \subset[T, X]^{r i}$. By Proposition 3.6. $T: \Lambda_{\Theta} \rightarrow X$ is not compact and hence $T:[T, X]^{r i} \rightarrow X$ is not compact either.

Remark 3.8. (a) In Proposition 3.6 the decreasing property of $t^{-1 / n^{\prime}} \varphi_{X}(t)$ is only needed to ensure "existence" of the space $\Lambda_{\Theta}$ and is not needed in the proof itself. Hence, Proposition 3.6 remains valid whenever $t^{-1 / n^{\prime}} \varphi_{X}(t)$ is decreasing in $(0, \delta)$ for some $0<\delta \leq 1$, [8, Remark 4.11]. The same comment applies to Theorem 3.7. With more generality, it suffices to have $t^{-1 / n^{\prime}} \varphi_{X}(t)$ "essentially decreasing" in the sense that it is equivalent to a decreasing function, that is, there exists a decreasing function $\psi$ and constants $0<C_{1}, C_{2}$ such that $C_{1} \psi(t) \leq t^{-1 / n^{\prime}} \varphi_{X}(t) \leq C_{2} \psi(t)$, for every $0<t \leq 1$.

(b) Whenever $t^{-1 / n^{\prime}} \varphi_{X}(t)$ is decreasing, we have $X \subset L^{n^{\prime}, \infty}([0,1])$.

(c) Within the class of those r.i. spaces $X$ for which $T:[T, X]^{r i} \rightarrow X$ is noncompact, there is a large subclass for which $T$ already fails compactness on some smaller r.i. domain (namely, $\Lambda_{\Theta}$ ).

For "most" r.i. spaces which occur in classical analysis, the alternative to $t^{-1 / n^{\prime}} \varphi_{X}(t)$ being decreasing is that $\lim _{t \rightarrow 0^{+}} t^{-1 / n^{\prime}} \varphi_{X}(t)=0$.

Theorem 3.9. Let $X$ be an r.i. space. Then $[T, X]^{r i}=L^{1}([0,1])$ and $T:[T, X]^{r i} \rightarrow$ $X$ is compact if and only if $\lim _{t \rightarrow 0^{+}} t^{-1 / n^{\prime}} \varphi_{X}(t)=0$.

Proof. The kernel operator $T$ has a density $F_{X}:(0,1] \rightarrow X$ given by $F_{X}(t)=$ $t^{-1 / n^{\prime}} \chi_{[0, t]}$; see [8, Section 3]. We verify that $F_{X}((0,1])$ is relatively compact in $X$ if and only if $\lim _{t \rightarrow 0^{+}} t^{-1 / n^{\prime}} \varphi_{X}(t)=0$. The proof of [8, Proposition 3.1(a)] shows that $F_{X}$ is continuous on $(0,1]$ with $\left\|F_{X}(t)\right\|_{X}=t^{-1 / n^{\prime}} \varphi_{X}(t)$. So, if

$$
\lim _{t \rightarrow 0^{+}} t^{-1 / n^{\prime}} \varphi_{X}(t)=0
$$

then $F_{X}(0):=0$ makes $F_{X}$ continuous on $[0,1]$. Hence, $F_{X}([0,1])$ is a compact set in $X$. Conversely, if $\left(t_{k}\right) \rightarrow 0^{+}$, then, for a subsequence (again denoted in the same way), $F_{X}\left(t_{k}\right)$ converges to some $g \in X$. Since $F_{X}\left(t_{k}\right)$ converges to zero a.e., it follows that $g=0$, [3, I.1.7]. This implies $\left\|F_{X}\left(t_{k}\right)\right\|_{X} \rightarrow 0$. Hence, $\lim _{t \rightarrow 0^{+}} t^{-1 / n^{\prime}} \varphi_{X}(t)=0$.

The condition $\lim _{t \rightarrow 0^{+}} t^{-1 / n^{\prime}} \varphi_{X}(t)=0$ implies that $\varphi_{X}(t) \leq C t^{1 / n^{\prime}}$ and so $L^{n^{\prime}, 1}([0,1]) \subset \Lambda_{X}$. Thus $L^{n^{\prime}, 1}([0,1]) \subset X$ and, by Remark 3.5, $[T, X]^{r i}=L^{1}([0,1])$. Hence, $T: L^{1}([0,1]) \rightarrow X$. Since $t^{-1 / n^{\prime}} \varphi_{X}(t) \leq C$, also $L^{1}([0,1]) \subset L^{1}\left(t^{-1 / n^{\prime}} \varphi_{X}(t)\right)$. So, the density $F_{X}$ of $T$ is Bochner integrable, [8, Proposition 3.4(b)]. The conclusion follows from the classical result that $T: L^{1}([0,1]) \rightarrow X$ is compact if and only if it has a Bochner integrable density whose range is essentially relatively compact in $X$, [10, VI.8.11].

Remark 3.10. For any r.i. space $X$, the inclusion $L^{n^{\prime}, 1}([0,1]) \subset X$ holds if and only if $L^{n^{\prime}, \infty}([0,1]) \subset M_{X}$ holds. The proof of Theorem 3.9 shows that the condition $\lim _{t \rightarrow 0^{+}} t^{-1 / n^{\prime}} \varphi_{X}(t)=0$ suffices for these conditions to hold. 
For $X=L^{p}([0,1])$, the condition of Theorem 3.7 is satisfied whenever $p \geq n^{\prime}$, so that $T:[T, X]^{r i} \rightarrow X$ is noncompact. The condition of Theorem 3.9 is satisfied for $p<n^{\prime}$, so that $T:[T, X]^{r i} \rightarrow X$ is compact in this case. For Lorentz spaces $L^{p, q}([0,1])$ a similar conclusion holds, namely $T:[T, X]^{r i} \rightarrow X$ is noncompact if and only if $p \geq n^{\prime}$. The Zygmund space $X=L_{\exp }$ is a Marcinkiewicz space with fundamental function $\varphi_{X}(t)=\log ^{-1}(e / t)$. Since $t^{-1 / n^{\prime}} \varphi_{X}(t)$ is decreasing, $T:\left[T, L_{\exp }\right]^{r i} \rightarrow L_{\exp }$ is noncompact (cf. Theorem 3.7). For the Zygmund space $X=L \log L$, we have $[T, L \log L]^{r i}=L^{1}([0,1])$ (see Remark [3.5) with $\varphi_{X}(t)=$ $t \log (e / t)$ and hence, $\lim _{t \rightarrow 0^{+}} t^{-1 / n^{\prime}} \varphi_{X}(t)=0$. By Theorem 3.9. $T:[T, L \log L]^{r i} \rightarrow$ $L \log L$ is compact. These classical spaces are included in the following larger class of r.i. spaces $X$ for which compactness of $T:[T, X]^{r i} \rightarrow X$ can be determined.

Example 3.11. Let $X$ be a Lorentz-Zygmund space $L^{p, q}(\log L)^{\alpha}$. If either $1<$ $p<\infty, 1 \leq q \leq \infty$ and $\alpha \in \mathbb{R}$, or $p=q=1$ and $\alpha \geq 0$, or $p=q=\infty$ and $\alpha<0$, then $X$ is an r.i. Banach space, [2], 3], [13]. As discussed in 2, this class contains many important spaces arising in classical analysis. Direct calculation shows:

(i) If either $p<n^{\prime}$, or $p=n^{\prime}$ and $\alpha<0$, then $\lim _{t \rightarrow 0^{+}} t^{-1 / n^{\prime}} \varphi_{X}(t)=0$.

(ii) If either $p>n^{\prime}$, or $p=n^{\prime}$ and $\alpha \geq 0$, then $t^{-1 / n^{\prime}} \varphi_{X}(t)$ is decreasing in $(0, \delta)$ for some $0<\delta \leq 1$.

So, for case (i) the operator $T:[T, X]^{r i} \rightarrow X$ is compact (cf. Theorem 3.9) and for case (ii) it is not (cf. Theorem 3.7 and Remark 3.8(a)).

Remark 3.12. For spaces $X$ as in Example 3.11(ii), Example 3.2 yields $\left[T, \Lambda_{X}\right]^{r i}=$ $\Lambda_{\Theta_{X}}$, where $\Theta_{X}(t)=t^{1 / n} \varphi_{X}(t)$. This can be used to explicitly determine further examples of optimal r.i. domains. For instance, if $X$ is a Lorentz $\Lambda$-space $L^{n^{\prime}, 1}(\log L)^{\alpha}$ with $\alpha \in \mathbb{R}$, then $\Theta_{X}$ is equivalent to $\Psi(t)=t \log ^{\alpha}(e / t)$. The space $\Lambda_{\Psi}$ is precisely the Zygmund space $L(\log L)^{\alpha}$, 3. IV.6.12], and hence,

$$
\left[T, L^{n^{\prime}, 1}(\log L)^{\alpha}\right]^{r i}=L(\log L)^{\alpha} .
$$

Note that $\alpha=1$ corresponds to the space $L \log L$.

The importance of the Zygmund spaces Exp $L^{p}$, consisting of functions having $p$-th exponential integrability $(p>0)$, [3, IV.6.11], warrants some comments concerning the space $\left[T, \operatorname{Exp} L^{p}\right]^{r i}$. The class $\operatorname{Exp} L^{p}$, for $p>0$, consists of spaces which are "close" to $L^{\infty}([0,1])$; it includes $L_{\exp }$ (i.e. $p=1$ ) and also the Orlicz space associated to $\phi(t)=\exp \left(t^{n^{\prime}}\right)-1$ (i.e. $p=n^{\prime}$ ) which appears in Trudinger's results in 20]. As Lorentz-Zygmund spaces these are given by $L^{\infty, \infty}(\log L)^{-1 / p}$, 2. Theorem 10.3]. Observe that $\operatorname{Exp} L^{p}$ are also Marcinkiewicz spaces but, since they fail to satisfy the index conditions of Example 3.3. [T, Exp $\left.L^{p}\right]^{r i}$ cannot be identified via Example 3.3.

The fundamental function of $\left[T, \operatorname{Exp} L^{p}\right]^{r i}$ is $\Psi(t):=t^{1 / n} \log ^{-1 / p}(e / t)$. To see this, it suffices to show that

$$
\Lambda_{\Psi} \subset\left[T, \operatorname{Exp} L^{p}\right]^{r i} \subset M_{\bar{\Psi}},
$$

for $\bar{\Psi}(t)=t / \Psi(t)$. Since, for $X=\operatorname{Exp} L^{p}$, the function

$$
t^{-1 / n^{\prime}} \varphi_{X}(t)=t^{-1 / n^{\prime}} \log ^{-1 / p}(e / t)
$$

is decreasing near zero (see Remark 3.8(a)), the first inclusion in (3.1) follows from Remark 2.1(b). To see that $\left[T, \operatorname{Exp} L^{p}\right]^{r i} \subset M_{\bar{\Psi}}$, let $f \in\left[T, \operatorname{Exp} L^{p}\right]^{r i}$. Then 
$f^{*} \in\left[T, \operatorname{Exp} L^{p}\right]$ and so $T f^{*} \in \operatorname{Exp} L^{p}$. Hence, for some constant $C>0$ and $0<t \leq 1$ we have

$$
\begin{aligned}
C t \log ^{1 / p}(e / t) & \geq \int_{0}^{t} T f^{*}(s) d s \\
& =\int_{0}^{t} f^{*}(x) x^{1 / n} d x+t \int_{t}^{1} f^{*}(x) x^{-1 / n^{\prime}} d x \\
& \geq \frac{n}{n+1} f^{*}(t) t^{(1 / n)+1} .
\end{aligned}
$$

Accordingly, $f^{*}(t) t^{1 / n} \log ^{-1 / p}(e / t) \leq C$ which implies, by (2.1), that $f \in M_{\bar{\Psi}}$. So (3.1) is established.

Precisely where the space $\left[T, \operatorname{Exp} L^{p}\right]^{r i}$ lies between $\Lambda_{\Psi}$ and $M_{\bar{\Psi}}$ is unclear. Since $\Lambda_{\Psi}=L^{n, 1}(\log L)^{-1 / p}$ and $M_{\bar{\Psi}}=L^{n, \infty}(\log L)^{-1 / p}$, we have from (3.1) that

$$
L^{n, 1}(\log L)^{-1 / p} \subset\left[T, \operatorname{Exp} L^{p}\right]^{r i} \subset L^{n, \infty}(\log L)^{-1 / p} .
$$

So, given $p>0$, a natural candidate for $\left[T, \operatorname{Exp} L^{p}\right]^{r i}$ is one of the spaces $L^{n, q}(\log L)^{-1 / p}$, which satisfy $\Lambda_{\Psi} \subset L^{n, q}(\log L)^{-1 / p} \subset M_{\bar{\Psi}}$ for every $1<q<\infty$, [2. Theorem 9.3]. However, none of these spaces coincide with $\left[T, \operatorname{Exp} L^{p}\right]^{r i}$. To see this choose $1 / q<\gamma<1$ and note that $g(t):=t^{-1 / n} \log ^{(1 / p)-\gamma}(e / t)$ belongs to $L^{n, q}(\log L)^{-1 / p}$. But, $g \notin\left[T, \operatorname{Exp} L^{p}\right]^{r i}$ since

$$
\begin{aligned}
\|T g\|_{\operatorname{Exp} L^{p}} & =\sup _{0<t \leq 1}\left(\log ^{-1 / p}(e / t)\right) \frac{1}{t} \int_{0}^{t}(T g)^{*}(s) d s \\
& \geq \sup _{0<t \leq 1} \log ^{-1 / p}(e / t) \int_{t}^{1} \frac{d x}{x \log ^{\gamma-(1 / p)}(e / x)} \\
& \approx \sup _{0<t \leq 1} \log ^{1-\gamma}(e / t)=\infty .
\end{aligned}
$$

A similar argument as above (with $\gamma=0$ ) shows that $\left[T, \operatorname{Exp} L^{p}\right]^{r i} \neq M_{\bar{\Psi}}$. It also turns out that $\left[T, \operatorname{Exp} L^{p}\right]^{r i} \neq \Lambda_{\Psi}=L^{n, 1}(\log L)^{-1 / p}$. Indeed, for $p \geq n^{\prime}$ Theorem 1 of [15] shows that $L^{n, p^{\prime}}([0,1])$ is included in $\left[T, \operatorname{Exp} L^{p}\right]^{r i}$. But, $L^{n, p^{\prime}}([0,1])$ is not included in $L^{n, 1}(\log L)^{-1 / p}$, as seen by considering a suitable function $h$ which, near zero, coincides with $t \mapsto t^{-1 / n} \log ^{-1 / p^{\prime}} \log \log (e / t)$. For $p<n^{\prime}$ Theorem 1 of [14] shows that $L^{n, n}(\log L)^{-\sigma / n}$ is included in $\left[T, \operatorname{Exp} L^{p}\right]^{r i}$, where $\sigma:=(n / p)-\left(n / n^{\prime}\right)$. However, $L^{n, n}(\log L)^{-\sigma / n}$ is also not included in $L^{n, 1}(\log L)^{-1 / p}$, which can be shown by considering the same function $h$ as above.

In particular, for $p=n^{\prime}$ the above facts imply that

$$
L^{n, 1}(\log L)^{-1 / n^{\prime}} \varsubsetneqq\left[T, \operatorname{Exp} L^{n^{\prime}}\right]^{r i} \varsubsetneqq L^{n, \infty}(\log L)^{-1 / n^{\prime}} .
$$

It is worthwhile to compare (3.2) with other results in the literature which deduce exponential integrability of a function from integrability properties of its gradient. According to the Introduction, $\left[T, \operatorname{Exp} L^{p}\right]^{r i}(\Omega)$ is the largest r.i. space having the property that a function $u: \Omega \rightarrow \mathbb{R}$ belongs to $\operatorname{Exp} L^{p}(\Omega)$, that is,

$$
\int_{\Omega} \exp (\lambda|u(x)|)^{p} d x<\infty
$$

for some $\lambda>0$, whenever $\nabla u \in\left[T, \operatorname{Exp} L^{p}\right]^{r i}(\Omega)$. To simplify what follows, set $Y_{1}=L^{n, p^{\prime}}(\Omega), Y_{2}=L^{n, n}(\log L)^{-\sigma / n}(\Omega)$ and $Y_{3}=L^{n, 1}(\log L)^{-1 / p}(\Omega)$. Let $p \geq n^{\prime}$. 
If $|\nabla u| \in Y_{1}$, that is

$$
\int_{0}^{1}\left(|\nabla u|^{*}(t) t^{1 / n}\right)^{1 / p^{\prime}} \frac{d t}{t}<\infty
$$

then (3.3) holds, [15. Accordingly, $Y_{1} \subset\left[T, \operatorname{Exp} L^{p}\right]^{r i}(\Omega)$. For $p<n^{\prime}$ it is shown in [14] that if

$$
\int_{\Omega}|\nabla u(x)|^{n} \log ^{-\sigma}(e+|\nabla u(x)|) d x<\infty,
$$

then (3.3) holds; this condition is equivalent to $|\nabla u| \in Y_{2}$, [2, Corollary 10.2]. Hence, $Y_{2} \subset\left[T, \operatorname{Exp} L^{p}\right]^{r i}(\Omega)$. Given any $0<p<\infty$, it follows from (3.2) that (3.3) holds whenever $|\nabla u| \in Y_{3}$, that is, whenever

$$
\int_{0}^{1}|\nabla u|^{*}(t) t^{1 / n} \log ^{-1 / p}(e / t) \frac{d t}{t}<\infty .
$$

So, also $Y_{3} \subset\left[T, \operatorname{Exp} L^{p}\right]^{r i}(\Omega)$.

The three proper subspaces $Y_{1}, Y_{2}$ and $Y_{3}$ of $\left[T, \operatorname{Exp} L^{p}\right]^{r i}(\Omega)$ are not comparable. Nevertheless, it is possible to formulate quantitatively "how different" these three spaces are from $\left[T, \operatorname{Exp} L^{p}\right]^{r i}(\Omega)$ by calculating the norm (in each space) of $\chi_{E}$, for any measurable set $E \subset \Omega$ having measure $a \in(0,1)$ for some fixed $a$. Indeed, $\left\|\chi_{E}\right\|_{Y_{1}}=a^{1 / n}$ and $\left\|\chi_{E}\right\|_{Y_{2}}=a^{1 / n} \log ^{\left(1 / n^{\prime}\right)-(1 / p)}(e / a)$ and $\left\|\chi_{E}\right\|_{Y_{3}}=$ $a^{1 / n} \log ^{-1 / p}(e / a)$. The last norm is the smallest one and, from (3.2), can be seen to coincide with the norm of $\chi_{E}$ in $\left[T, \operatorname{Exp} L^{p}\right]^{r i}(\Omega)$.

\section{Compactness properties of the Sobolev imbedding}

Our main focus in this section is on the $X(\Omega)$-valued imbedding $j$, always assumed to be acting on its optimal Sobolev domain $W_{0}^{1}[T, X]^{r i}(\Omega)$. In Theorem 4.2 it is established that $j$ fails to be compact whenever $t^{-1 / n^{\prime}} \varphi_{X}(t)$ is decreasing on $(0, \delta)$ for some $0<\delta \leq 1$. Under this condition, the theorem actually states more: namely, noncompactness of $j$ already occurs for the space $W_{0}^{1} \Lambda_{\Theta}(\Omega)$, where $\Lambda_{\Theta}$ (with $\left.\Theta(t):=t^{1 / n} \varphi_{X}(t)\right)$ is typically smaller than $[T, X]^{r i}$. In Theorem 4.4, compactness of $j$ is established under the condition $\lim _{t \rightarrow 0^{+}} t^{-1 / n^{\prime}} \varphi_{X}(t)=0$.

Theorem 4.1. Let $X$ and $Y$ be r.i. spaces such that $Y \subset[T, X]^{r i}$. If $T: Y \rightarrow X$ is noncompact, then the Sobolev imbedding $j: W_{0}^{1} Y(\Omega) \hookrightarrow X(\Omega)$ is bounded, but not compact.

Proof. Since $Y \subset[T, X]^{r i}$, the operator $T: Y \rightarrow X$ is bounded. By the results of Edmunds, Kerman and Pick mentioned in the Introduction, $j: W_{0}^{1} Y(\Omega) \hookrightarrow X(\Omega)$ is bounded.

Since $T: Y \rightarrow X$ is noncompact, there exists $\varepsilon>0$ and a sequence $\left(f_{k}\right)$ with $\left\|f_{k}\right\|_{Y} \leq 1$ such that $\left\|T f_{k}-T f_{k^{\prime}}\right\|_{X} \geq \varepsilon$, for every $k \neq k^{\prime}$. We may assume that $f_{k} \geq 0$. Indeed, if $B_{Y}$ denotes the unit ball of $Y$ and $B_{Y}^{+}$its positive part, then $T\left(B_{Y}\right) \subset T\left(B_{Y}^{+}\right)-T\left(B_{Y}^{+}\right)$. Since $T\left(B_{Y}\right)$ is not relatively compact in $X$, neither is $T\left(B_{Y}^{+}\right)$.

Claim. Let $f \in Y$ with $\|f\|_{Y} \leq 1$ and $f \geq 0$. There exists $u \in W_{0}^{1} Y(\Omega)$ with $u \geq 0$, such that $u^{*}=T f$ and $\|u\|_{W_{0}^{1} Y(\Omega)} \leq M$, where the constant $M$ does not depend on $f$. 
Assuming the Claim for now, there exist functions $u_{k} \in W_{0}^{1} Y(\Omega)$ with $u_{k} \geq 0$, such that $u_{k}^{*}=T f_{k}$ and $\left\|u_{k}\right\|_{W_{0}^{1} Y(\Omega)} \leq M$, for $k \in \mathbb{N}$. By boundedness of the imbedding $j$, we have $\left(u_{k}\right) \subset X(\Omega)$. By a theorem of Lorentz and Shimogaki, [3. III.7.4 and II.4.6], if $Z$ is an r.i. space and $f, g \in Z$ with $f, g \geq 0$, then $\|f-g\|_{Z} \geq\left\|f^{*}-g^{*}\right\|_{Z}$. So, for $k \neq k^{\prime}$, we have

$$
\begin{aligned}
\left\|u_{k}-u_{k^{\prime}}\right\|_{X(\Omega)} & =\left\|\left(u_{k}-u_{k^{\prime}}\right)^{*}\right\|_{X} \geq\left\|u_{k}^{*}-u_{k^{\prime}}^{*}\right\|_{X} \\
& =\left\|T f_{k}-T f_{k^{\prime}}\right\|_{X} \geq \varepsilon .
\end{aligned}
$$

This implies that $j: W_{0}^{1} Y(\Omega) \hookrightarrow X(\Omega)$ is noncompact.

To verify the Claim let $f \in Y$ satisfy $\|f\|_{Y} \leq 1$ and $f \geq 0$. We follow the construction in the proof of Theorem 3.8 in 11 to define a function $u: \Omega \rightarrow \mathbb{R}$, supported in a ball $B\left(x_{0}, R\right) \subset \Omega$, by $u(x):=\int_{K\left|x-x_{0}\right|^{n}}^{1} f(t) t^{-1 / n^{\prime}} d t$, for some constant $K>0$. Then $u \geq 0, u^{*}=T f$ and $|\nabla u|^{*}$ is a multiple of $D_{\alpha} f^{*}$, for some $\alpha>0$, where $D_{\alpha}$ is the dilation operator corresponding to $\alpha$. Since $T$ maps $L^{1}$ into $L^{1}$ and $L^{\infty}$ into $L^{\infty}$ and $Y$ is r.i., $T$ maps $Y$ into itself. So, $u^{*}=T f \in Y$ with $\|u\|_{Y(\Omega)}=\left\|u^{*}\right\|_{Y} \leq\|T\|_{Y \rightarrow Y}$. Since $Y$ is r.i. and $D_{\alpha}$ maps $Y$ into itself, we have $D_{\alpha} f^{*}=\left(D_{\alpha} f\right)^{*} \in Y$ and so $|\nabla u|^{*} \in Y$ with $\left\|\left.\left|\nabla u\left\|_{Y(\Omega)}=\right\|\right| \nabla u\right|^{*}\right\|_{Y} \leq$ $C\left\|D_{\alpha}\right\|_{Y \rightarrow Y}$. By a regularization argument, $u \in W_{0}^{1} Y(\Omega)$ and $\|u\|_{W_{0}^{1} Y(\Omega)} \leq M$.

Theorem 4.2. Let $X$ be an r.i. space with $t^{-1 / n^{\prime}} \varphi_{X}(t)$ decreasing. For $\Theta(t):=$ $t^{1 / n} \varphi_{X}(t)$ the imbedding $j: W_{0}^{1} \Lambda_{\Theta}(\Omega) \hookrightarrow X(\Omega)$ is noncompact. Hence, also the Sobolev imbedding

$$
j: W_{0}^{1}[T, X]^{r i}(\Omega) \hookrightarrow X(\Omega)
$$

fails to be compact.

Proof. By Proposition 3.6. $T: \Lambda_{\Theta} \rightarrow X$ is noncompact. Remark 2.1(b) shows that $\Lambda_{\Theta} \subset[T, X]^{r i}$. Applying Theorem 4.1 we see that $j: W_{0}^{1} \Lambda_{\Theta}(\Omega) \hookrightarrow X(\Omega)$ is noncompact. Since $\Lambda_{\Theta} \subset[T, X]^{r i}$, the imbedding $j: W_{0}^{1} \Lambda_{\Theta}(\Omega) \hookrightarrow X(\Omega)$ factors through $W_{0}^{1}[T, X]^{r i}(\Omega)$. Accordingly, $j: W_{0}^{1}[T, X]^{r i}(\Omega) \hookrightarrow X(\Omega)$ is noncompact either.

Remark 4.3. (a) Theorem 4.2 is also valid under the local condition on $\varphi_{X}$ given in Remark 3.8(a).

(b) For $p=1$, the Rellich-Kondrachov theorem implies that $W_{0}^{1} L^{1}(\Omega) \hookrightarrow$ $L^{n^{\prime}}(\Omega)$ is noncompact. The largest r.i. space $X$ to which Theorem 4.2 applies is $L^{n^{\prime}, \infty}([0,1])$. For this case, $j: W_{0}^{1} L^{1}(\Omega) \hookrightarrow L^{n^{\prime}, \infty}(\Omega)$ is noncompact (by Remark 3.5), with $L^{n^{\prime}, \infty}(\Omega)$ being larger than $L^{n^{\prime}}(\Omega)$.

(c) For $X=L^{p}([0,1])$ with $n^{\prime} \leq p<\infty$ the Rellich-Kondrachov theorem implies that $j: W_{0}^{1} L^{p_{0}}(\Omega) \hookrightarrow L^{p}(\Omega)$ fails to be compact, where $p_{0}=n p /(n+p)$. By Theorem $4.2 j: W_{0}^{1} L^{p_{0}, 1}(\Omega) \hookrightarrow L^{p}(\Omega)$ is noncompact, with $L^{p_{0}, 1}(\Omega)$ being smaller than $L^{p_{0}}(\Omega)$.

(d) For $X=L^{p, \infty}([0,1])$ with $n^{\prime}<p<\infty$, Theorem 4.2 and Example 3.3 give that $j: W_{0}^{1} L^{p_{0}, \infty}(\Omega) \hookrightarrow L^{p, \infty}(\Omega)$ is noncompact, where $p_{0}=n p /(n+p)$.

We now consider compactness of the imbedding $j$.

Theorem 4.4. Let $X$ be an r.i. space with $\lim _{t \rightarrow 0^{+}} t^{-1 / n^{\prime}} \varphi_{X}(t)=0$. Then $[T, X]^{r i}=L^{1}([0,1])$, and we have compactness of the Sobolev imbedding

$$
j: W_{0}^{1} L^{1}(\Omega) \hookrightarrow X(\Omega) .
$$


Proof. Since $\lim _{t \rightarrow 0^{+}} t^{-1 / n^{\prime}} \varphi_{X}(t)=0$, Remark 3.10 shows that $L^{n^{\prime}, 1}([0,1]) \subset X$. So, $[T, X]^{r i}=L^{1}([0,1])$ by Remark [3.5, Let $B$ be the unit ball of $W_{0}^{1} L^{1}(\Omega)$. By a standard reduction argument it suffices to assume that $B$ is the unit ball of $C_{0}^{1}(\Omega)$, relative to the norm from $W_{0}^{1} L^{1}(\Omega)$. Then, as in the proof of the RellichKondrachov theorem, an argument based on the Ascoli-Arzelá Theorem (e.g. 21, p. 62]) shows that $B$ is relatively compact in $L^{1}(\Omega)$ hence, relatively compact in measure.

Since $\left[T, L^{n^{\prime}, 1}\right]=L^{1}([0,1])$, we have $j: W_{0}^{1} L^{1}(\Omega) \hookrightarrow L^{n^{\prime}, 1}(\Omega)$ is bounded. Hence, $B$ is bounded in $L^{n^{\prime}, 1}(\Omega)$. Moreover, $L^{n^{\prime}, 1}(\Omega) \subset X(\Omega)$ implies that $B$ is contained in $X(\Omega)$. We claim $B$ has equi-absolutely continuous norm in $X(\Omega)$, that is, given $\varepsilon>0$ there exists $\delta>0$ such that if $m(A)<\delta$, then $\left\|f \chi_{A}\right\|_{X(\Omega)}<\epsilon$ for every $f \in B$. Since $B$ is bounded in $L^{n^{\prime}, 1}(\Omega)$, it suffices to show that the unit ball of $L^{n^{\prime}, 1}(\Omega)$ has this property. So, let $f \in L^{n^{\prime}, 1}(\Omega)$ with $\|f\|_{n^{\prime}, 1} \leq 1$. Then

$$
\begin{aligned}
\left\|f \chi_{A}\right\|_{X(\Omega)} & \leq\left\|f \chi_{A}\right\|_{\Lambda_{X}(\Omega)}=\int_{0}^{1}\left(f \chi_{A}\right)^{*}(t) \varphi_{X}^{\prime}(t) d t \\
& \leq \int_{0}^{m(A)} f^{*}(t) \varphi_{X}^{\prime}(t) d t \leq\|f\|_{n^{\prime}, 1}\left\|\varphi_{X}^{\prime} \chi_{[0, m(A))}\right\|_{n, \infty} \\
& \leq \sup _{0<t \leq 1}\left(\varphi_{X}^{\prime} \chi_{[0, m(A))}\right)^{*}(t) t^{1 / n}=\sup _{0<t \leq m(A)} \varphi_{X}^{\prime}(t) t^{1 / n} \\
& \leq \sup _{0<t \leq m(A)} \varphi_{X}(t) t^{-1 / n^{\prime}},
\end{aligned}
$$

where the second inequality follows from $\int_{0}^{t}\left(f_{1} f_{2}\right)^{*} \leq \int_{0}^{t} f_{1}^{*} f_{2}^{*}$, valid for every $0 \leq t \leq 1$, [3, p. 88], and from Hardy's Lemma, 3, II.3.6], applicable since $\varphi_{x}^{\prime}$ is decreasing. The final inequality follows from $\varphi_{X}^{\prime}(t) \leq \varphi_{X}(t) / t$, since $\varphi_{X}$ is quasiconcave. So, if $m(A) \rightarrow 0$, then $\left\|f \chi_{A}\right\|_{X(\Omega)} \rightarrow 0$ uniformly for $f$ in the unit ball of $L^{n^{\prime}, 1}(\Omega)$.

Now, $B$ is relatively compact in measure and has equi-absolutely continuous norm in $X(\Omega)$. Adapting the proof in [16, Theorem 11.3] (written for Orlicz norms), we deduce that $B$ is relatively compact in $X(\Omega)$. Hence, $j: W_{0}^{1} L^{1}(\Omega) \hookrightarrow X(\Omega)$ is compact.

Remark 4.5. (a) Since $L^{1}([0,1])$ is the largest possible r.i. space, under the condition of Theorem 4.4 we have compactness of the Sobolev imbedding $j: W_{0}^{1} Y(\Omega) \hookrightarrow$ $X(\Omega)$ for every r.i. space $Y$.

(b) For $X=L^{p}([0,1])$ with $1 \leq p<n^{\prime}$, the Rellich-Kondrachov theorem gives compactness of $j: W_{0}^{1} L^{1}(\Omega) \hookrightarrow X(\Omega)$. This case is also included in Theorem 4.4 since $t^{-1 / n^{\prime}} \varphi_{X}(t)=t^{(1 / p)-\left(1 / n^{\prime}\right)} \rightarrow 0$ as $t \rightarrow 0^{+}$. Actually, if $X$ is any r.i. space for which there exists $1 \leq p<n^{\prime}$ such that $L^{p}([0,1]) \subset X$, then the RellichKondrachov theorem also implies compactness of $j: W_{0}^{1} L^{1}(\Omega) \hookrightarrow X(\Omega)$. However, the Marcinkiewicz space $X$ with fundamental function $\varphi_{X}(t)=t^{1 / n^{\prime}} \log (e / t)$ is an example of an r.i. space to which Theorem 4.4 applies, yet $L^{p}([0,1])$ is not contained in $X$ for any $1 \leq p<n^{\prime}$.

(c) Donaldson and Trudinger studied compactness of the Sobolev imbedding $W_{0}^{1} L_{B}(\Omega) \hookrightarrow L_{A}(\Omega)$ where $L_{B}$ and $L_{A}$ are Orlicz spaces; see [9] and [1, Theorem 8.32]. The case $L_{B}=L^{1}([0,1])$ is a priori excluded $\left(L^{1}([0,1])\right.$ is not an Orlicz space). However, the proof remains valid showing that $j: W_{0}^{1} L^{1}(\Omega) \hookrightarrow$ 
$L_{A}(\Omega)$ is compact whenever $A(t)$ increases at infinity "more slowly" than $t^{n^{\prime}}$; see [1, §8.5]. This is equivalent to $\lim _{x \rightarrow \infty} x^{1 / n^{\prime}} / A^{-1}(x)=0,[1, \S 8.5]$, that is, to $\lim _{t \rightarrow 0^{+}} t^{-1 / n^{\prime}} \varphi_{L_{A}}(t)=0$ by [3, IV.8.17]. This is precisely the condition of Theorem 4.4

Example 4.6. Let $X$ be one of the Lorentz-Zygmund spaces $L^{p, q}(\log L)^{\alpha}$ of Example 3.11, It follows from that example and Theorem 4.2 that the imbedding $j: W_{0}^{1}[T, X]^{r i}(\Omega) \hookrightarrow X(\Omega)$ is noncompact if either $p>n^{\prime}$ or $p=n^{\prime}$ and $\alpha \geq 0$, and from that example and Theorem 4.4 that $j$ is compact if either $p<n^{\prime}$ or $p=n^{\prime}$ and $\alpha<0$.

Our final result (a consequence of Theorems 3.9 and 4.4) highlights, once again, the role of the kernel operator $T:[T, X]^{r i} \rightarrow X$.

Theorem 4.7. Let $X$ be an r.i. space for which $[T, X]^{r i}=L^{1}([0,1])$. If $T:[T, X]^{r i}$ $\rightarrow X$ is compact, then so is the Sobolev imbedding

$$
j: W_{0}^{1} L^{1}(\Omega) \hookrightarrow X(\Omega) .
$$

\section{REFERENCES}

[1] R. A. Adams, Sobolev Spaces, Academic Press, Boston, 1978. MR0450957 (56:9247)

[2] C. Bennett and K. Rudnick, On Lorentz-Zygmund spaces, Dissert. Math. 175 (1980), 1-67. MR0576995 (81i:42020)

[3] C. Bennett and R. Sharpley, Interpolation of Operators, Academic Press, Boston, 1988. MR0928802 (89e:46001)

[4] Yu. A. Brudnyı̆ and N. Ya. Krugljak, Interpolation Functors and Interpolation Spaces, NorthHolland, Amsterdam, 1991. MR1107298 (93b:46141)

[5] A. Cianchi and L. Pick, Sobolev embeddings into BMO, VMO, and $L_{\infty}$ spaces, Ark. Mat. 36 (1998), 317-340. MR1650446 (99k:46052)

[6] G. P. Curbera, When $L^{1}$ of a vector measure is an $A L$-space, Pacific J. Math. 162 (1994), 287-303. MR.1251903 (94k:46070)

[7] G. P. Curbera and W. J. Ricker, Optimal domains for kernel operators via interpolation, Math. Nachr. 244 (2002), 47-63. MR1928916 (2003g:47045)

[8] G. P. Curbera and W. J. Ricker, Optimal domains for the kernel operator associated with Sobolev's inequality, Studia Math. 158 (2003) 131-152 and 170 (2005), 217-218. MR2013736 (2005b:46068)

[9] T. K. Donaldson and N. S. Trudinger, Orlicz-Sobolev spaces and imbedding theorems, J. Funct. Anal. 8 (1971), 52-75. MR0301500 (46:658)

[10] N. S. Dunford and J. T. Schwartz, Linear Operators, Vol. I, Wiley-Interscience, New York, 1958. MR0117523(22:8302)

[11] D. Edmunds, R. Kerman and L. Pick, Optimal Sobolev imbeddings involving rearrangementinvariant quasinorms, J. Funct. Anal. 170 (2000), 307-355. MR.1740655 (2000m:46070)

[12] D. Edmunds, B. Opic and L. Pick, Poincaré and Friedrichs inequalities in abstract Sobolev spaces, Math. Proc. Cambridge Philos. Soc. 113 (1993), 355-379. MR.1198418 (94e:46057)

[13] W. D. Evans, B. Opic and L. Pick, Interpolation of operators on scales of generalized LorentzZygmund spaces, Math. Nachr. 182 (1996), 127-181. MR1419893 (97m:46041)

[14] N. Fusco, P. L. Lions and C. Sbordone, Sobolev imbedding theorems in borderline cases, Proc. Amer. Math. Soc. 124 (1996), 561-565. MR1301025 (96d:46035)

[15] S. Hudson and M. Leckband, A sharp exponential inequality for Lorentz-Sobolev spaces on bounded domains, Proc. Amer. Math. Soc. 127 (1999), 2029-2033. MR1643410 (99j:46036)

[16] M. A. Krasnosel'skii and Ya. B. Rutickii, Convex Functions and Orlicz Spaces, Noordhoff, Gröningen, 1961. MR0126722 (23:A4016)

[17] S. G. Krein, Ju. I. Petunin and E. M. Semenov, Interpolation of Linear Operators, Amer. Math. Soc., Rhode Island, 1982. MR0649411 (84j:46103)

[18] D. R. Lewis, On integrability and summability in vector spaces, Illinois J. Math., 16 (1972), 294-307. MR0291409(45:502) 
[19] L. Pick, Optimal Sobolev Embeddings, Rudolph-Lipschitz-Vorlesungsreihe Nr. 43, SFB 256: Nichtlineare Partielle Differentialgleichungen, University of Bonn, 2002.

[20] N. S. Trudinger, On imbeddings into Orlicz spaces and some applications, J. Math. Mech. 17 (1967), 473-484. MR0216286 (35:7121)

[21] W. P. Ziemer, Weakly Differentiable Functions, Springer-Verlag, New York, 1989. MR1014685 (91e:46046)

Facultad de Matemáticas, Universidad de Sevilla, Aptdo. 1160, Sevilla 41080, Spain

E-mail address: curbera@us.es

Math.-Geogr. Fakultät, Katholische Universität Eichstätt-Ingolstadt, D-85072 EICHStÄTt, GERMANY

E-mail address: werner.ricker@ku-eichstaett.de 